Proceeding of the International Conference on Advanced Science,
Engineering and Information Technology 2011

\title{
A Bi-directional Energy Splitable Model for Energy Optimization in Wireless Sensor Networks
}

\author{
A Rajeswari ${ }^{1}$, P T Kalaivaani ${ }^{2}$ \\ 1-Department of Electronics and Communication Engineering, \\ Coimbatore Institute of Technology - 641014, Tamilnadu, India \\ Tel.:+91-9865907804, E-mail: rajeswari.ece.cit@gmail.com \\ 2-Department of Electronics and Communication Engineering, \\ Sri Krishna College of Engineering and Technology- 641008, Tamilnadu, India \\ Tel.:+91-9003321111, E-mail: kalaivani.p.t@gmail.com
}

\begin{abstract}
Wireless Sensor Networks is a budding prototype of networking and computing, where a node may be self powered and individual node have the capability to sense and compute and communicate. Wireless Sensor Networks have been proposed for variety of applications such as Industrial control and monitoring and home automation and consumer electronics and security and Military sensing, Asset tracking and supply chain management, Intelligent Agriculture, Missile directing, Fire alarming, Landslide Warning, Environmental monitoring and health monitoring and commercial applications. In Wireless Sensor Network large number of nodes are deployed randomly. Depends on the network architecture the application may be personalized such as Energy Efficiency, Routing and Power Management and data dissemination. Energy Optimization involves in minimizing an energy expenditure and maximizing the lifetime of the complete network. In the proposed work, the placement of nodes are directly involved with residual energy. Energy Optimization in sensor network is very difficult task to achieve it. The optimization of energy is performed through Bidirectional Energy Splitable Model . The data flow in both forward and backward directions are considered, In order to achieve the best QOS in transmission, some parameters such as load, delay and direction of individual nodes are considered. A mathematical model is developed to determine the data flow of individual node based on the residual energy.
\end{abstract}

Keywords: Wireless Sensor Networks, Energy Optimization, Bi-directional Energy Splitable Model (BES), Residual Energy

\section{INTRODUCTION}

In the design aspect of wireless sensor network major challenge is energy optimization. A well planned node placement in a network prototype is an effective way to achieve the goal of energy constraint. A sensor network is composed of a large number of sensor nodes that are densely deployed either inside the phenomenon or very close to it. The position of individual sensor nodes need not be predetermined. Nodes available in a sensor network prone to failures. Very often, the topology of a sensor network changes. Broadcast communication paradigm is adapted mainly in Wireless Sensor Networks. In a Wireless
Sensor Network, the network protocols and algorithms must possess self - organizing capabilities. Co-operative effort of sensor nodes is another inimitable feature of sensor networks. Sensor nodes are limited in power and memory and computational competence. Sensors are positioned in an unreceptive environment and recharging is almost impossible during the operation. So energy optimization or energy efficient solutions are needed to increase the lifetime of a whole wireless sensor network [14].

Energy efficiency in sensor networks can be achieved through several techniques. Single Link and Multi-Link energy efficient forwarding and Spatial 
Correlation method, Protocols such as LEACH, LEACH-C, GEAR, SPIN (Sensor Protocols for Information via Negotiation), MAC based protocols such as CC-MAC, Self Organizing Medium Access control for Sensor Networks (SMACS), Eaves drop and register (EAR) algorithm and Cooperative Communication Scheme and relaying scheme.

Cross Layer Heuristic Approach, eSENSE, Dynamic Voltage Scaling, Data filtering and Link Adaptation Technique and Placement of the nodes are some of the methods to achieve Energy Optimization in sensor networks.

In this paper, Bi-directional Energy Splitable (BES) model is proposed and the performance is analyzed with Bidirectional model. BES model is useful to maximize the lifetime of the network through the forward and backward dataflow considerations. The proposed BES model yields better results compare to that of bi-directional model. If the space deployment of nodes are random or even spaced, it is difficult to achieve the longer lifetime but when the nodes in a sensor network are spaced according to their residual energy then it leads to achieve much longer life time.

\section{RELATED WORKS}

Enrico Natalizio et al., investigated the deployment of wireless nodes for maximizing the lifespan of the network [1]. The mathematical model is developed to identify the best placement of nodes and drawback of the work is that the model provides best result only in single data flow. Stavros Toumpis et al., proposed massively dense network that is useful to avoid the congestion [2]. Ivan Stojmenovic et al., proposed a routing protocol that minimizes the energy required per routing task and the communication overhead. Node mobility with proper location update scheme is the main objective. Major drawback of the work is that the network is best suited with high connectivity and all the concepts are demand based one [3]. Enrico Natalizio et al., investigated the placement of nodes based on the residual energy. QOS parameter needs to be optimized for to identify the lifespan of the network [4]. Zhen jiang et al., proposed the solutions to control the relay nodes in WSN. These solutions are useful to obtain the optimal in local coordinate systems. The drawback associated in the work is distance from the current position to the target position need to be larger always [5]. The above discussed literature survey is a part of paper [14]. Yunxia Chen et al., proposed greedy approach to enhance the lifetime of individual nodes. MAC protocol that exploits both the channel state information and residual energy of individual sensors. For each data collection, the protocol maximizes the minimum residual energy across the network. A general formula for network lifetime which holds independently of underlying network model is achieved [11]. Mehmet C.Vuran, Ian F. Akyildiz proposed spatial correlation in wireless sensor network on MAC layer. They proposed a theoretical framework for transmission regulation of sensor networks under a distortion constraint [12].

\section{PERFORMANCE OF ENERGY OPTIMIZATION THROUGH BES MODEL}

Each node in a sensor network integrated with sensors, processors, transceivers with limited resources and low capacity battery is associated with each node. Primary source of energy to sensor node is battery. To achieve the longer lifetime in WSN, each node organize themselves for the purpose of sensing their field of view, and pass some information to some users.

At regular interval of time, the nodes available in a sensor network collects the data points and transform all the data points into an equivalent electric signal and distribute the signal to the sink or base node via some reliable communication medium. Each node collects some raw data and send it to central node and performs the computation to take final decision. Sensor nodes are spatially distributed in nature and the ambient conditions related to surrounding environment of the sensors are measured by sensing circuitries in sensor network. Generally two different types of processing is available in sensor network. They are Non coherent and coherent. In Non coherent processing, raw data will be preprocessed at each node to extract a set of parameters where as in coherent processing, raw data will be tagged with timestamp and uploaded to a central node. Elements of wireless sensor network may be homogeneous or heterogeneous. Homogeneous elements are more reliable with low cost and heterogeneous elements are accomplished with increased functionality than homogeneous elements. Sensor networks allow new nodes to be added and reconfigure when existing nodes fail. Data dissemination is classified into two types they are Multicast and Unicast. The SPIN, Flooding protocols are the category of Multicast data dissemination and LEACH, TEEN, SAR and directed diffusion type protocols comes under the category of Unicast data dissemination. Nodes are deployed randomly. After the deployment, nodes are stationary. [14]

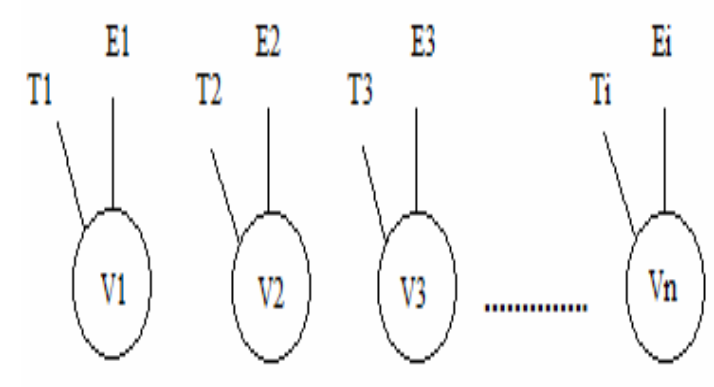

Fig. 1 Network Model

Consider a Wireless Sensor Network with ' $n$ ' number of nodes from $V_{1}$ to $V_{n}$. Each node is associated with certain number of relay nodes. Let $T_{i}$ and $E_{i}$ be the lifetime and residual energy of the each nodes respectively. Minimum required power in the receiver is denoted by $\mathrm{P}_{\mathrm{rec}}$. According to this transmitter can adjust its power level to achieve the proper delivery of $\mathrm{P}_{\mathrm{rec}}$ in the receiver. 
Mathematical formulation of bi-directional energy splitable model is given by

$$
T \leq \frac{E \times \lambda}{P_{\text {rec }}\left\|V_{n}-V_{n-1}\right\|^{2}}
$$

Where $\mathrm{T}$ is the function of distance between the current node and to the neighbor node [1]. $\lambda$ is a function flow parameter. Flow parameter is considered as a function of load, delay. Depending on the value of $\lambda$, the best placement of node can be achieved. Flow parameter is given by the equation [2],

$$
\lambda=L+D
$$

A central management station is available in which all the node's residual energy and position information are updated regularly. Central Management Station is required to collect all the updated information.

This paper presents a solution to optimize the energy using Bi-directional Energy splitable Model and energy optimization is done in a better way.

In BES model, central management station monitors the individual node's flow parameter, load, delay and their residual energy value. The delay of a network specifies how long it takes for a bit of data to travel across the network from one node to another. It is typically measured in multiples or fractions of seconds. Delay may differ slightly, depending on the location of the specific pair of communicating nodes. Load is an important criteria, it should be distributed evenly across two or more nodes. Flow parameter is the combination of load and delay. The value of flow parameter and node number are automatically generated in this consideration. Depending on the flow parameter, the residual energy of the device is split between the two directions. The nodes which have less value of flow parameter , (ex: $\lambda=0.10 .2,0.3)$ are moving in forward directions. The nodes which have high value of flow parameter (ex: $\lambda=0.7,0.8)$ are moving in backward direction. Residual energy of each node is calculated from the movement of direction. To perform residual energy calculation, the node number is monitored continuously along with flow parameter. When the flow parameter value is decreased to lower value immediately the node number is noted then corresponding node's load and delay parameter value is taken to perform analysis. Depending on the node direction, the energy usage is calculated. If the node is moved in forward direction the energy usage is less, else it consumes much energy to move in backward side. The flow parameter value is an important criteria to decide node direction and to calculate the energy value also. In the proposed scheme $\lambda$ value is considered from 0.1 to 0.9 . BES model with $\lambda=0.1$ is closest to the first node in forward direction, symmetrically $\lambda=0.9$ is closest to the first node in backward direction. Various values of load and delay for the given set of nodes and their corresponding direction are tabulated.[14]
TABLE I

Flow Parameter Vs Node Direction

\begin{tabular}{|c|c|c|c|c|}
\hline $\begin{array}{c}\text { Node } \\
\text { number }\end{array}$ & Delay & Load & $\boldsymbol{\lambda}$ & $\begin{array}{c}\text { Node } \\
\text { direction }\end{array}$ \\
\hline 1 & .1 & .2 & 0.3 & Forward \\
\hline 2 & .2 & .2 & 0.4 & Forward \\
\hline 3 & .2 & .3 & 0.5 & Central \\
\hline 4 & .3 & .3 & 0.6 & Backward \\
\hline 5 & .4 & .3 & 0.7 & Backward \\
\hline 6 & .5 & .4 & 0.9 & Backward \\
\hline
\end{tabular}

In table II and III the delay value is kept as constant. In table II, the constant delay value maintained throughout the work is 0.1 and in Table III, the constant delay value maintained is 0.2 .Corresponding to delay value the flow parameter value is calculated

TABLE II

Flow Parameter Vs Node Direction With the Constant Delay Value 0.1

\begin{tabular}{|c|c|c|c|c|}
\hline $\begin{array}{c}\text { Node } \\
\text { Number }\end{array}$ & Delay & Load & $\boldsymbol{\lambda}$ & $\begin{array}{c}\text { Node } \\
\text { direction }\end{array}$ \\
\hline 1 & 0.1 & 0.1 & 0.2 & Forward \\
\hline 2 & 0.1 & 0.2 & 0.3 & Forward \\
\hline 3 & 0.1 & 0.3 & 0.4 & Forward \\
\hline 4 & 0.1 & 0.4 & 0.5 & Central \\
\hline 5 & 0.1 & 0.5 & 0.6 & Backward \\
\hline 6 & 0.1 & 0.6 & 0.7 & Backward \\
\hline 7 & 0.1 & 0.7 & 0.8 & Backward \\
\hline 8 & 0.1 & 0.8 & 0.9 & Backward \\
\hline 9 & 0.1 & 0.9 & 1 & Backward \\
\hline
\end{tabular}

TABLE III

Flow Parameter Vs Node Direction With the Constant Delay Value 0.2

\begin{tabular}{|c|c|c|c|c|}
\hline $\begin{array}{c}\text { Node } \\
\text { Number }\end{array}$ & Delay & Load & $\lambda$ & $\begin{array}{c}\text { Node } \\
\text { direction }\end{array}$ \\
\hline 1 & 0.2 & 0.1 & 0.3 & Forward \\
\hline 2 & 0.2 & 0.2 & 0.4 & Forward \\
\hline 3 & 0.2 & 0.3 & 0.5 & Central \\
\hline 4 & 0.2 & 0.4 & 0.6 & Backward \\
\hline 5 & 0.2 & 0.5 & 0.7 & Backward \\
\hline 6 & 0.2 & 0.6 & 0.8 & Backward \\
\hline 7 & 0.2 & 0.7 & 0.9 & Backward \\
\hline 8 & 0.2 & 0.8 & 1 & Backward \\
\hline 9 & 0.2 & 0.9 & 1.1 & Backward \\
\hline
\end{tabular}


Flow Parameter Vs Node Number

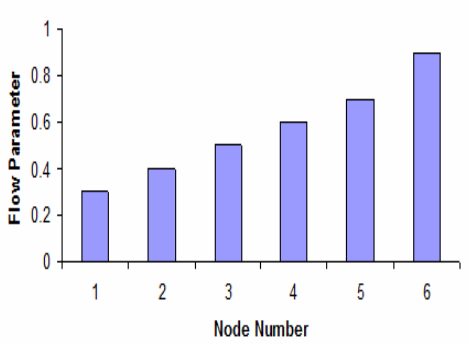

Fig. 2 Flow Parameter Vs Node number

Fig. 2 shows set of values of flow parameter and its corresponding node number. Here the total number of node consideration is six.

Flow Parameter Vs Node Number

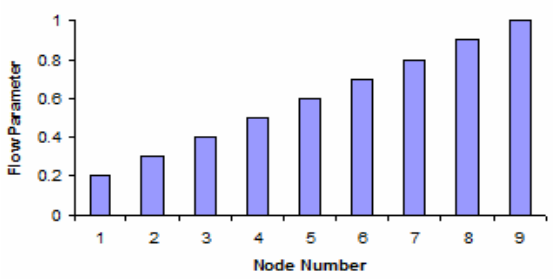

Fig. 3 Flow Parameter Vs Node number

Similarly in Fig.3 also different set of values of flow parameter and the corresponding node number values are plotted. Totally nine nodes are considered. When the load and delay value changes automatically the flow parameter value is changed for each individual node.

Fig.4 indicates the analysis of BES model when $\lambda$ varies in terms of path life time. When Energy is split in two directions, $\lambda=0.5$, the longest life time is achieved. Lifespan is decreased when $\lambda$ is less than 0.5 and $\lambda$ is greater than 0.5 . When the values of $\lambda$ varies other than 0.5 , in order to give priority to a direction over the other, BES model allows to maximize the path-lifetime by determining the individual nodes placement.

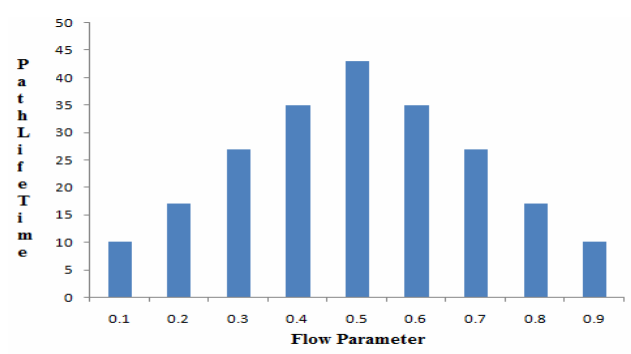

Fig .4 Flow Parameter Vs Path Life Time
A significant improvement in lifetime of the network and minimization of energy can be achieved through the factors node position, node direction and flow parameter. Monodirectional and bi-directional models are useful to identify the node's position but in the case of bidirectional energy splitable model (BES), individual node position is identified along with their residual energy. The proposed system not only improves the lifetime of the network but also minimizes the energy based on the node position and node direction. In BES model, energy is calculated according to that the node direction in forward and backward movement is identified. BES model is useful to calculate the longest path lifetime.

In future work, the identical concept can be adapted to wireless sensor networks in real time environment with large number of nodes. The same idea can be implemented to sensor networks with precomputed environment.

\section{REFERENCES}

[1] E.Natalizio, V.Loscri, and E.Viterbo "Optimal Placement of Wireless Nodes for Maximizing Path Lifetime" in IEEE Communication Lett.,vol.12.no.5,pp.362-364, May 2008

[2] S.Toumpis and L.Tassiulas, "Optimal Deployment of Large Wireless Sensor Networks" IEEE Trans Inform.Theory,vol.52,no.7,pp.29352953, July 2006

[3] I.Stojmenovic and X.Lin, " Power-aware Localized Routing in Wirelessnetworks" IEEE Trans.Parallel and Distrib.syst., vol.12,no.11,pp.1122 - 1133, Nov .2001

[4] Enrico Natalizio,Valeria Loscri,Francesca Guerriero,Anonio Violi "Energy Spaced Placement for Bidirectional Data Flows in Wireless Sensor Networks" IEEE Communication Let.,vol13,N0.1, Jan 2009

[5] Z.Jiang,J.Wu and R.Kline "Mobility Control for Achieving Optimal Configuration in Wireless Sensor Networks," in Wireless Networks, Springer Netherlands, June 2008

[6] X.Cheng,Z.jiang,ad J.wu,"Mobility Control Schemes with Quick Convergence in Wireless Sensor Networks" in proc.10, International Workshop on advances in parallel and distributed computational Models, 2008

[7] Jason A. Fuemmeler,Venugopal V. Veeravalli," STmart Sleeping Policies for Energy Efficient Tracking in Sensor Networks," IEEE Transactions on signal processing, vol. 56, no. 5, May 2008

[8] Ritesh Madan, Shuguang Cui, Sanjay Lall, Andrea J. Goldsmith , "Modeling and Optimization of Transmission Schemes in EnergyConstrained Wireless Sensor Networks" IEEE/ACM Transactions on Networking, vol. 15, no. 6, Dec 2007

[9] Shuguang Cui , Jin-Jun Xiao, Andrea J. Goldsmith , Zhi-Quan Luo , H.Vincent Poor "Estimation Diversity and Energy Efficiency in Distributed Sensing” IEEE Transactions on Signal Processing, vol. 55, no. 9 , Sept 2007

[10] D.W.Carman, B.J.Matt "Energy-efficient and Low-latency Key Management For Sensor Networks" Communications Security Architecture for Army Sensor Networks, Sept 2000.

[11] Yunxia Chen, Qing Zhao "On the Lifetime of Wireless Sensor Networks" IEEE Communication Letters, vol.9, N0..11, Nov 2005

[12] Mehmet C.Vuran, Ian F. Akyildiz "Spatial Correlation-Based Collaborative Medium Access Control in Wireless Sensor Network", IEEE / ACM Transactions on Networking, Vol.14, No.2, Apr 2006.

[13] Hnin Yu Shwe, JIANG Xiao-hong, Susumu Horiguchi “Energy Saving in Wireless Sensor Networks" Journal of Communication and Computer, volume 6, No.5 (Serial No.54), ISSN 1548-7709, USA, May 2009

[14] A.Rajeswari , P T Kalaivaani "Energy Optimization in Wireless Sensor Network Using Bidirectional Energy Splitable Model”, in the Proceedings of National Conference on Emerging Trends in Wireless Technologies (ETWT-2010), pp-113-116, July 2010. 